\title{
COMPLICAÇÕES ORAIS EM PACIENTES EM TRATAMENTO QUIMIOTERÁPICO NA UNACON, NO MUNICÍPIO DE ITAJAÍ/SC
}

\author{
ORAL MANIFESTATION IN PATIENTS DURING \\ CHEMOTHERAPY TREATMENT AT UNACON, ITAJAÍ/SC
}

Maria Regina Orofino Kreuger

LuANa WolfF SAVOLd

SCHeila HofFmanN

Nivaldo Murilo Diegoli
Professora Doutora da Disciplina de Patologia Geral Aplicada dos Cursos de Odontologia, Farmácia e Medicina da Universidade do Vale de Itajaí - SC (Univali)

Cirurgiã-dentista egressa do Curso de Odontologia da Universidade do Vale do Itajaí - SC (Univali)

Cirurgiã-dentista egressa do Curso de Odontologia da Universidade do Vale do Itajaí - SC (Univali)

Professor Mestre das Disciplinas de Metodologia Científica e Trabalho de Conclusão de Curso dos Cursos de Odontologia e Medicina da Universidade do Vale do Itajaí - SC (Univali)

Fonte Financiadora: Programa de Iniciação Científica Artigo 170/Governo do Estado de SANTA Catarina/Univali

\section{RESUMO}

As lesões na cavidade oral compreendem as mais frequentes complicações da quimioterapia antineoplásica, devido à alta sensibilidade das estruturas orais aos efeitos tóxicos dos quimioterápicos. $\mathrm{O}$ objetivo deste trabalho foi registrar as complicações orais mais comuns em pacientes submetidos à quimioterapia do câncer, relacioná-las ao sexo e à idade, determinando a frequência dessas lesões e a possível interação com a medicação quimioterápica em uso. Este trabalho foi realizado na Unidade de Alta Complexidade em Oncologia, no município de Itajaí/SC. Foram analisados os prontuários dos pacientes, registrando dados pessoais e aspectos referentes à patologia de cada um deles. Foi efetuado um exame clínico oral para avaliar as lesões desses pacientes. A tabulação dos dados foi efetuada por meio da distribuição de frequência relativa de cada tipo de lesão encontrada, segundo faixa etária, gênero e medicação utilizada. Os resultados foram: o gênero mais frequente foi o feminino $(60 \%)$; a idade mais afetada foi acima de 50 anos (57,8\%); o tumor mais frequente foi o de mama (11 casos); as manifestações orais encontradas foram xerostomia (46 casos), afta (dois casos), herpes (dois casos), candidíase (um caso) e mucosite (um caso). A maioria dos pacientes apresentou algum tipo de manifestação oral, sendo a xerostomia a mais frequente; as infecções orais mais encontradas foram a candidíase e a herpes; o maior número de pacientes foi do gênero feminino; a faixa etária mais prevalente foi acima de 50 anos em ambos os gêneros. A manifestação oral mais prevalente quanto à interação com os medicamentos foi a xerostomia.

Palavras-chave: CÂNCER - QUIMIOTERAPIA - MANIFESTACÕES ORAIS

\section{Abstract}

Chemotherapy affects cells with high mitotic index. Lesions in the oral cavity are the most frequent complication of antineoplastic chemotherapy, because of the high sensitivity of oral tissues and structures to the toxic effects of the chemotherapics. The objective of this work was to register the most common oral complications found in patients undergoing to cancer chemotherapy, determining the frequency of the lesion and the possible interaction with the medication in use. This work was conducted at CEPON (Oncological Center Care), in Itajaí (SC), Brazil. Patients' reports were analyzed and personal data, therapeutic plan and the type of the tumor information were registered. An oral clinical examination was performed to detect buccal manifestations. The data were registered by the distributing the frequency of each episode in accordance with age, gender, and therapeutic modality. The results showed that the most frequent gender was female (60\%); in both genders, the age was over 50 years (57,8\%); breast cancer was the most incident tumor (11 episodes); oral manifestations found were xerostomy (46 episodes), aphtha (2 episodes), herpes simplex (2 episodes) and candidiases (1 episode). In conclusion the females older than 50 years were the most affected. Most patients had some oral manifestation with a highest incidence of oral dryness, and it was related with many different chemotherapics, being a xerostomy-induced medication.

Keyword: CANCER - CHEMOTHERAPY - ORAL MANIFESTATIONS 
INTRODUÇÃo E REVISÃo DE LiterATURA

A quimioterapia consiste no uso de drogas injetáveis que provocam queda da imunidade, propiciando o aparecimento de lesões orais, que muitas vezes são desconhecidas pelo próprio paciente ou pelos familiares deste. Em razão disso, é necessário um acompanhamento odontológico mais próximo e possibilitando o tratamento medicamentoso dessas lesões.

De acordo com Naylor e Terezhalmy, ${ }^{1}$ a quimioterapia do câncer é usada para destruir rapidamente as células proliferativas. Porém, células normais com alta taxa mitótica também são afetadas por essa intervenção, particularmente aquelas que estão na mucosa oral, gastrointestinal e no sistema hematopoiético. Isso pode estar associado ao aparecimento de complicações orais, tais como: mucosites, infecções, hemorragias, xerostomia e desordens neurológicas e nutricionais. Dreizen ${ }^{2}$ relatou que nenhuma parte do corpo reflete as complicações do tratamento do câncer tão visivelmente quanto à boca. Os sinais infecciosos, hemorrágicos, citotóxicos, nutricionais e neurológicos da toxicidade das drogas são refletidos na boca pela mudança de cor, característica, conforto e continuidade da mucosa.

Conforme Mealey et al., ${ }^{3}$ dependendo dos tipos e dosagens dos agentes quimioterápicos usados, várias complicações orais podem surgir. As infecções decorrentes podem ser recorrentes e de difícil tratamento e, geralmente, são de caráter fúngico, pela Candida albicans.

De acordo com Sung, ${ }^{4}$ pelo crescente número de pacientes em tratamento quimioterápico, cerca de $40 \%$ desenvolverão problemas orais significantes durante a exposição ao medicamento. Pelo rápido desenvolvimento e ritmo de renovação das células, a mucosa oral é muito vulnerável à quimioterapia.
Rosa $^{5}$ relatou que os quimioterápicos são drogas que atuam principalmente sobre as células tumorais, destruindo-as ou impedindo a sua reprodução. Entretanto, algum dano sobre os tecidos normais é inevitável, particularmente naqueles em que ocorre naturalmente uma rápida divisão celular (cabelos, mucosas, sistema hematopoiético). Assim sendo, muitos desses medicamentos estão relacionados com complicações orais e podem produzir estomatotoxicidade direta ou indiretamente.

Montenegro e Franco ${ }^{6}$ afirmaram que aproximadamente 85.000 brasileiros morreram de câncer em 1991, sendo em torno de 45.200 homens e 38.300 mulheres; a região Sul do Brasil é onde há maior incidência de linfomas.

Brasileiro Filho ${ }^{7}$ relatou a prevalência dos principais tumores malignos nos homens e nas mulheres, no Brasil. Os mais frequentes nas mulheres são os de pele $(24,3 \%)$, seguido de colo de útero $(20,1 \%)$ e mama $(16,6 \%)$; entre os homens, os mais frequentes são os de pele $(28,7 \%)$, estômago $(12,2 \%)$ e próstata $(6,9 \%)$.

Para Castro et al., ${ }^{8}$ alguns dos problemas causados pela quimio e radioterapia são mucosite, hipossalivação, perda do paladar, perda do apetite, doença periodontal, cárie rampante e osteorradionecrose.

Segundo Martins et al., ${ }^{9}$ as complicações orais decorrentes da estomatotoxicidade direta (efeitos não específicos das drogas nas células em mitose) e indireta (efeitos em células específicas, como as da medula óssea, que propiciam o desenvolvimento de infecções e hemorragias) são: mucosite - alteração em todas as mucosas atingidas pela citotoxicidade dos oncoterápicos e que está presente entre $40 \%$ a $76 \%$ dos pacientes sob quimioterapia; xerostomia - causa dificuldade de deglutição, acúmulo de placa bacteriana que, associada a carboidratos, aumenta a incidência de cárie, 
neurotoxicidade, infecções bacterianas, fúngicas e virais e sangramento oral.

Jensen et al. ${ }^{10}$ afirmaram que a saliva tem funções importantes como solução tampão, defesa imunológica, equilíbrio da microbiota e proteção da mucosa, com consequente manutenção da integridade dos tecidos da boca. Pacientes que apresentam câncer e que se submetem ao tratamento frequentemente apresentam dificuldade para manter tais funções. Além disso, a deterioração da saúde oral e os sintomas orais incluindo a xerostomia têm influência significativa na qualidade de vida durante e após o tratamento dos pacientes. A quimioterapia causa mudanças na composição e no fluxo salivar durante o tratamento.

De acordo com Sandoval et al., ${ }^{11} \mathrm{o}$ tratamento oncológico frequentemente envolve o uso de radioterapia e/ou quimioterapia. Uma complicação comum desse tipo de terapia é a mucosite oral, que causa grande impacto na qualidade de vida do paciente durante o tratamento e, às vezes, pode levar à interrupção deste, resultando em graves consequências em termo da resposta tumoral. Aproximadamente $60 \%$ dos pacientes em tratamento radioterápico convencional de cabeça e pescoço e mais de $90 \%$ dos pacientes submetidos a uma terapia combinada (quimioterapia e radioterapia concomitantes) são sujeitos a desenvolvimento de grave mucosite oral. Até $40 \%$ dos pacientes submetidos a esse tratamento desenvolvem mucosite oral, principalmente quando o regime inclui fluorouracil-5, metotrexato e cisplatina.

Segundo Travaglini, ${ }^{12}$ aproximadamente uma semana ou 15 dias após a sessão de quimioterapia, o paciente entra em imunossupressão, que é a queda da resistência. Nesse período, qualquer foco de infecção odontogênica ou periodontal preexistente pode representar um grande risco de o paciente desenvolver infecções orais.

Guerra et al. $^{13}$ relataram que, de acordo com os dados de dez registros de câncer de base populacional do Brasil, os tumores mais frequentes no país são: próstata, pulmão, estômago, cólon, reto e esôfago na população masculina. Em mulheres, predomina o câncer de mama, seguido de colo uterino, cólon e reto, pulmão e estômago. Com relação ao sexo, a prevalência de câncer entre homens e mulheres é muito similar nos países desenvolvidos; nos países em desenvolvimento, a prevalência é $25 \%$ maior em mulheres, com um predomínio em homens de câncer com pior taxa de sobrevida, tais como fígado, esôfago e estômago.

Kumar et al. ${ }^{14}$ relataram que a idade tem uma influência importante na probabilidade de uma pessoa ser acometida por um câncer. A maioria dos carcinomas ocorre mais tardiamente na vida (acima de 55 anos).

Mendonça et al. ${ }^{15}$ afirmaram que não só o câncer oral produz alterações na boca, mas também a terapia utilizada para os diversos tipos de neoplasias malignas, por exemplo, quimioterapia e radioterapia. Esses tratamentos provocam alterações orais como: mucosite, xerostomia, neurotoxicidade, hipogeusia, trismo muscular, osteorradionecrose, sangramento gengival, candidose, herpes labial e queilite angular.

Para Santos e Fernandes, ${ }^{16}$ os fatores que afetam a frequência do desenvolvimento de problemas orais estão relacionados ao paciente e à terapia. Os fatores que se relacionam ao paciente são: idade, diagnóstico e a sua condição oral antes e durante a terapêutica. E os fatores relacionados à terapia são o tipo de droga quimioterápica, a dose e a frequência do tratamento.

Vieira e Lopes ${ }^{17}$ afirmaram que, na quimioterapia, o aparecimento da mucosite geralmente ocorre entre cinco a 
dez dias pós-tratamento e depende do tipo de fármaco administrado.

Napeñas et al. ${ }^{18}$ relataram que o mecanismo patofisiológico da mucosite inclui uma cascata de eventos destrutivos e inflamatórios iniciados por agentes citotóxicos e exacerbados pela presença de fatores locais e sistêmicos. Os fatores locais incluem mudanças no fluxo salivar, trauma nos tecidos locais e a presença de micro-organismos. A quimioterapia citotóxica compromete os mecanismos de defesa da boca fisicamente (danos diretos à mucosa) e imunologicamente (neutropenia) causando mudança na microflora oral. As bactérias orais podem contribuir para a mudança no processo patológico, e uma bactéria comensal pode se tornar patogênica se ela atingir a corrente sanguínea.

Considerando que a grande parte dos pacientes acometidos de lesões cancerígenas passará por tratamento antineoplásico, como a quimioterapia, a proposta desta pesquisa foi verificar as complicações orais em pacientes submetidos ao tratamento quimioterápico, o tipo de câncer que eles apresentam e correlacionar essas complicações com a idade, o sexo e a medicação quimioterápica.

\section{Materiais e Métodos}

Esta pesquisa se caracteriza como um estudo exploratório-descritivo, mediante coleta de dados primários e secundários realizada na Unidade de Alta Complexidade em Oncologia (Unacon) do Hospital e Maternidade Marieta Konder Bornhausen, no município de Itajaí/SC. Foi analisada a cavidade oral de pacientes dessa unidade, em tratamento quimioterápico para câncer, no período de abril a novembro de 2007.
A amostra, não probabilística, foi obtida por conveniência, com um total de 86 pacientes que, por livre e espontânea vontade, aceitaram participar da pesquisa cujo projeto foi previamente aprovado pela Comissão de Ética em Pesquisa da Universidade do Vale do Itajaí (Univali).

Por meio da análise dos prontuários, foram obtidos e registrados os dados pessoais (gênero e idade) e aqueles referentes à patologia e tratamento de cada paciente. Por intermédio de exame clínico, foram obtidos os dados referentes às manifestações orais.

O exame clínico foi realizado com o paciente no leito enquanto este realizava a sessão de quimioterapia. Cada paciente foi submetido a um questionário não estruturado acerca das alterações bucais. Após a entrevista, foi realizado o exame clínico com auxílio de espátula de madeira, luz artificial e materiais de biossegurança para as pesquisadoras, tais como: jaleco, óculos de proteção, máscara, luvas e gorro. Foi analisada a mucosa oral dos pacientes para detecção de qualquer comprometimento.

Os pacientes foram informados sobre as manifestações orais que poderiam ocorrer durante o tratamento e quais os cuidados necessários em relação a elas.

\section{Resultados e Discussão}

Foram realizadas 23 visitas à Unidade Oncológica do Hospital e Maternidade Marieta Konder Bornhausen, no período de abril a novembro de 2007. As consultas odontológicas foram realizadas no período matutino, totalizando 86 pacientes.

Quanto à distribuição do sexo dos pacientes atendidos, $60 \%$ foram do sexo feminino e $40 \%$, do sexo masculino (Gráfico 1). 
GrÁFICO 1 - DisTRIBUIÇÃO DA FREQUÊNCIA RELATIVA DA CATEGORIA GÊNERO DOS PACIENTES EM TRATAMENTO QUIMIOTERÁPICO NA UNACON DE ITAJAÍ SC.

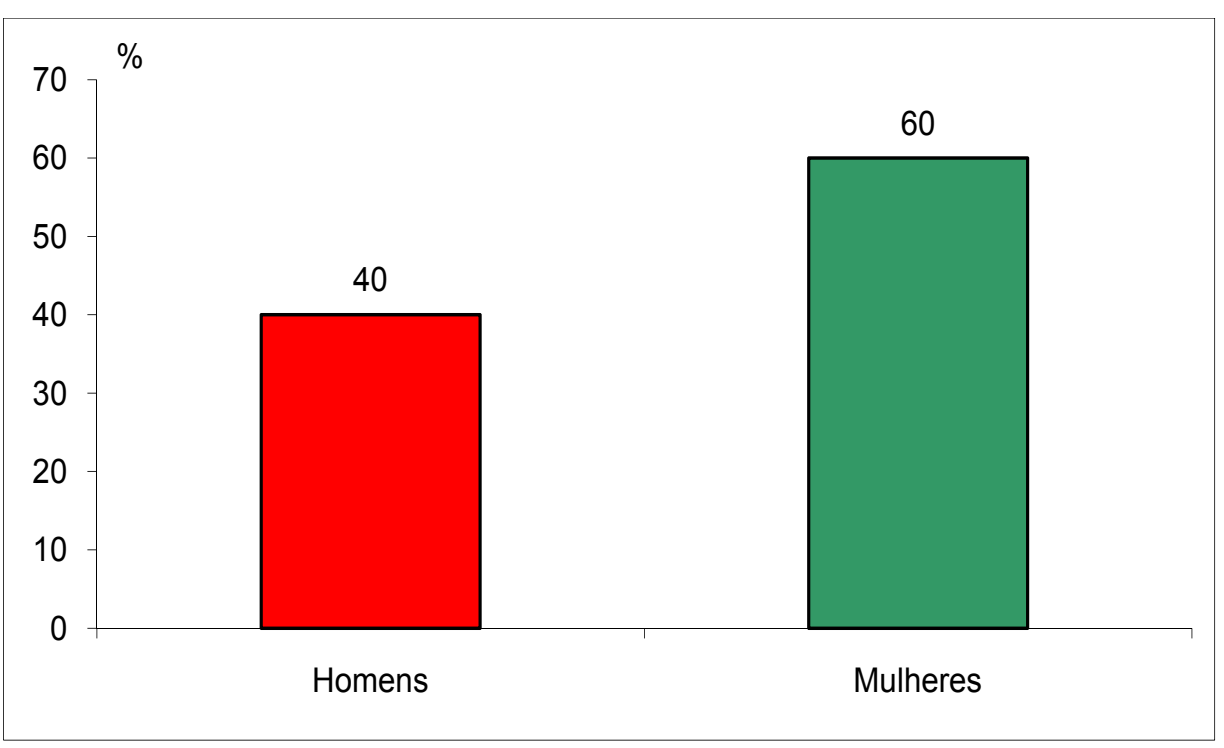

Conforme explicitado anteriormente, a prevalência de câncer entre homens e mulheres é muito similar nos países desenvolvidos, enquanto nos países em desenvolvimento a prevalência de mulheres é $25 \%$ maior, o que reflete o predomínio, em homens, de localizações de câncer com pior sobrevida, como fígado, esôfago e estômago (Guerra et al. ${ }^{13}$ ). O fato de nesta amostra ter ocorrido maior percentual de mulheres em tratamento, em virtude de chamadas anuais para exames preven- tivos de câncer ginecológico, possivelmente possibilitou diagnóstico precoce de alterações neoplásicas.

Para a determinação das faixas etárias mais frequentes nos pacientes em atendimento na Unacon, as idades destes foram agrupadas por décadas. A faixa etária mais prevalente foi a de 51 a 60 anos (24 pacientes). Verificou-se que $57,83 \%$ dos pacientes pesquisados incluem-se na faixa etária superior a 50 anos (Gráfico 2).

GrÁFICo 2 - DistribuIÇÃO DA FAIXA ETÁRIA PREVALENTE DOS PACIENTES EM TRATAMENTO QUIMIOTERÁPICO NA UNACON DE ITAJAí SC.

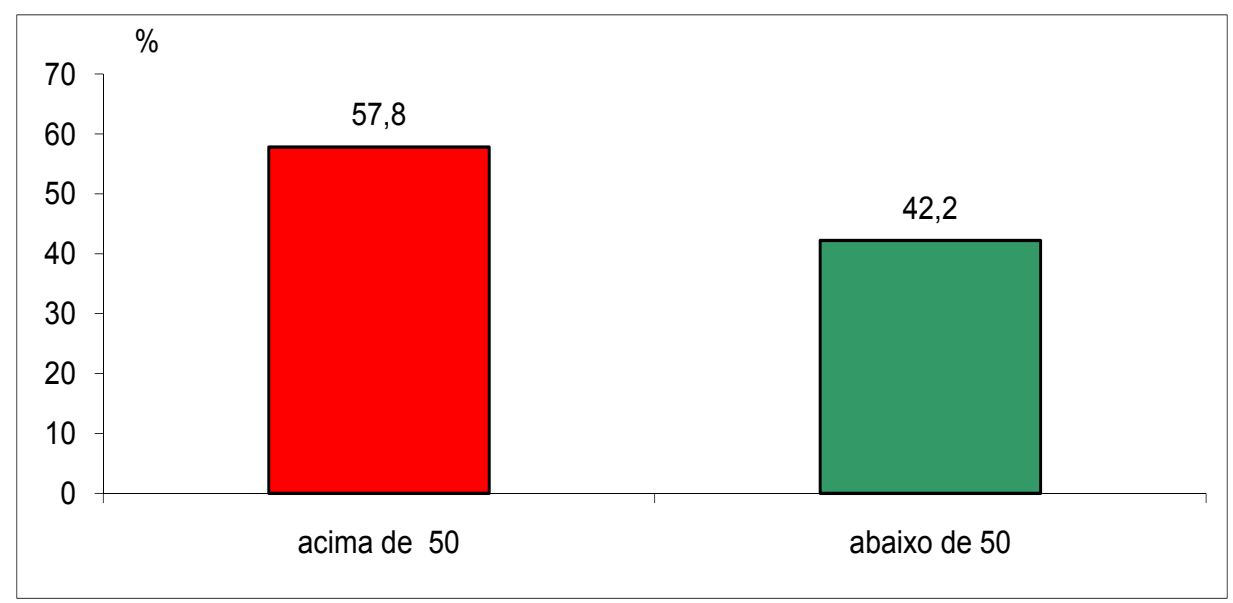


A idade tem importante influência na probabilidade do paciente adquirir câncer. A maioria dos carcinomas ocorre mais tardiamente, ou seja, em pessoas acima de 55 anos, porém, os dados estatísticos mais recentes trouxeram boas notícias: as taxas de morte ajustadas pela idade entre 1990 e 2000 caíram em ambos os sexos. ${ }^{14}$ Infelizmente, são muito escassos os estudos de incidência de câncer no nosso país, tendo em vista a inexistência de registros adequados da doença. Os dados sobre câncer no Brasil baseiam-se, em grande parte, na prevalência dos centros de diagnóstico e tratamento e, em parte, em registros sobre mortalidade. Esses registros indicam que ocorre um aumento expressivo da ocorrência de neoplasias com o avançar da idade, sendo a faixa de 60 a 79 anos a de maior incidência tanto em homens quanto em mulheres. ${ }^{7}$

As neoplasias primárias encontradas entre os pacientes variaram em torno de aproximadamente 21 tipos distintos, sendo que as de mama e linfomas foram as mais frequentes (Gráfico 3).

Gráfico 3 - DisTribuiÇÃo QUANTO AO LOCAL DAS NEOPLASIAS ENCONTRADAS NOS PACIENTES EM TRATAMENTO QUIMIOTERÁPICO NA UNACON DE ITAJAí SC

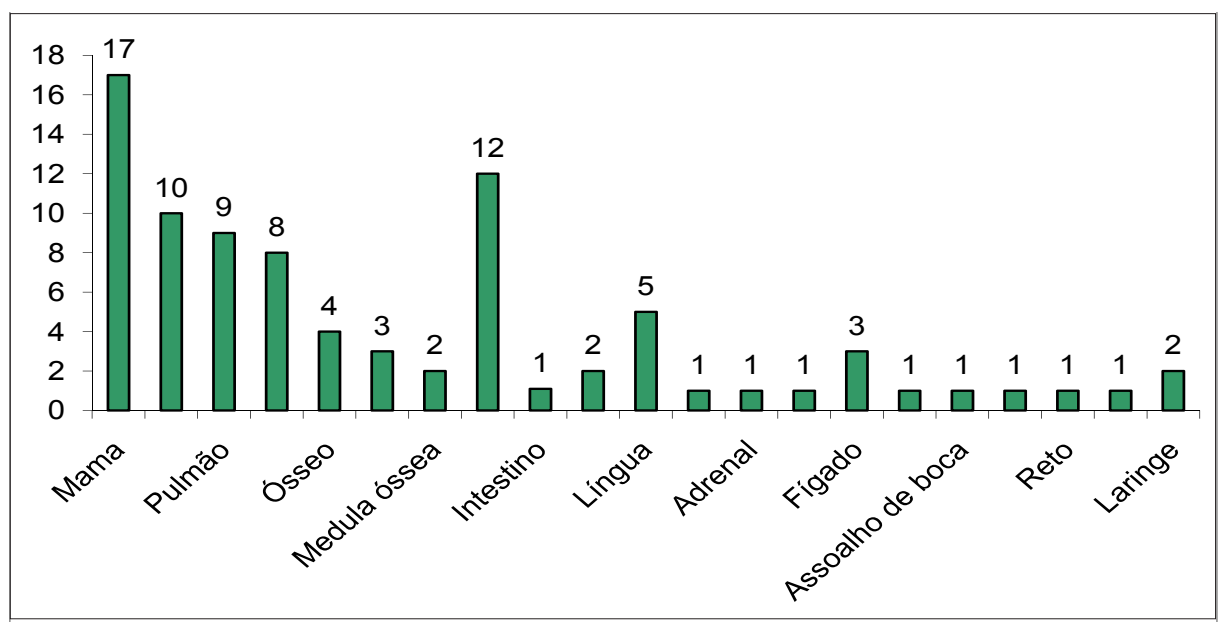

De acordo com os dados, de dez registros de câncer de base populacional do Brasil, os tumores mais frequentes são: próstata, pulmão, estômago, cólon e reto e esôfago na população masculina; em mulheres, predomina o câncer de mama, seguido de colo uterino, cólon e reto, pulmão e estômago. Vale destacar que, segundo a Organização Mundial da Saúde (2002), sobressaemse, entre os cinco tipos mais frequentes, os tumores de pulmão, de cólon e reto e de estômago, tanto nos países industrializados quanto nos países em desenvolvimento. ${ }^{13}$

O grande número de casos de câncer de mama em tratamento reflete um maior controle no aparecimento e tratamento dessa neoplasia, provavelmente pelos exames de mamografia realizados pelo sistema de saúde nacional, uma vez que esses exames são gratuitos. Os linfomas foram o segundo tipo de câncer mais encontrado neste trabalho. Montenegro e Franco ${ }^{6}$ relataram que a região Sul do Brasil é onde os linfomas são mais incidentes, isso pode explicar a amostra encontrada neste trabalho em relação a esse tipo de neoplasia.

Dos 86 pacientes examinados, $56 \%$ apresentaram alguma alteração na cavidade oral (Gráfico 4). A manifestação mais frequente foi a xerostomia (Gráfico 5). 
GrÁFICO 4 - DisTRIBUIÇÃo EM RELAÇÃo À PRESENÇA DE MANIFESTAÇÕES ORAIS DOS PACIENTES EM TRATAMENTO QUimioterÁPICO NA UNACON DE ITAJAI SC.

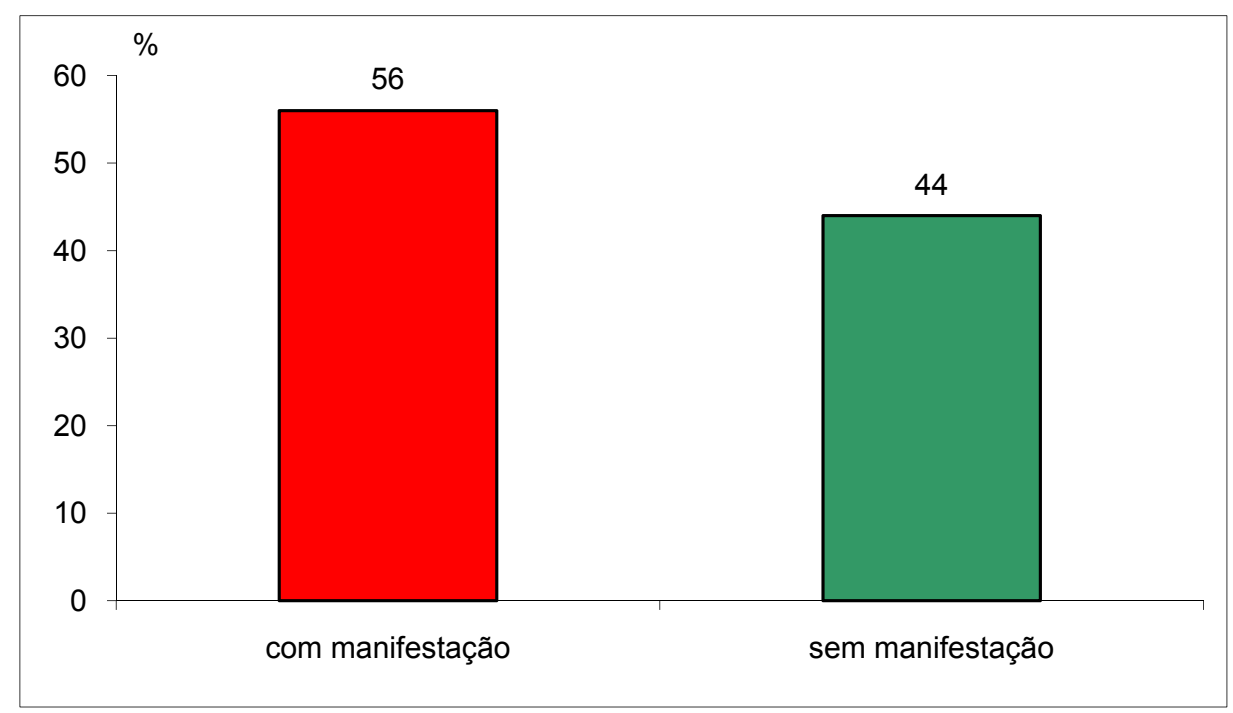

Gráfico 5 - Distribuição DAS MANIFESTAÇÕES ORAIS APRESENTADAS PELOS PACIENTES EM TRATAMENTO QUIMIOTERÁPICO NA UNACON DE ITAJAÍ SC.

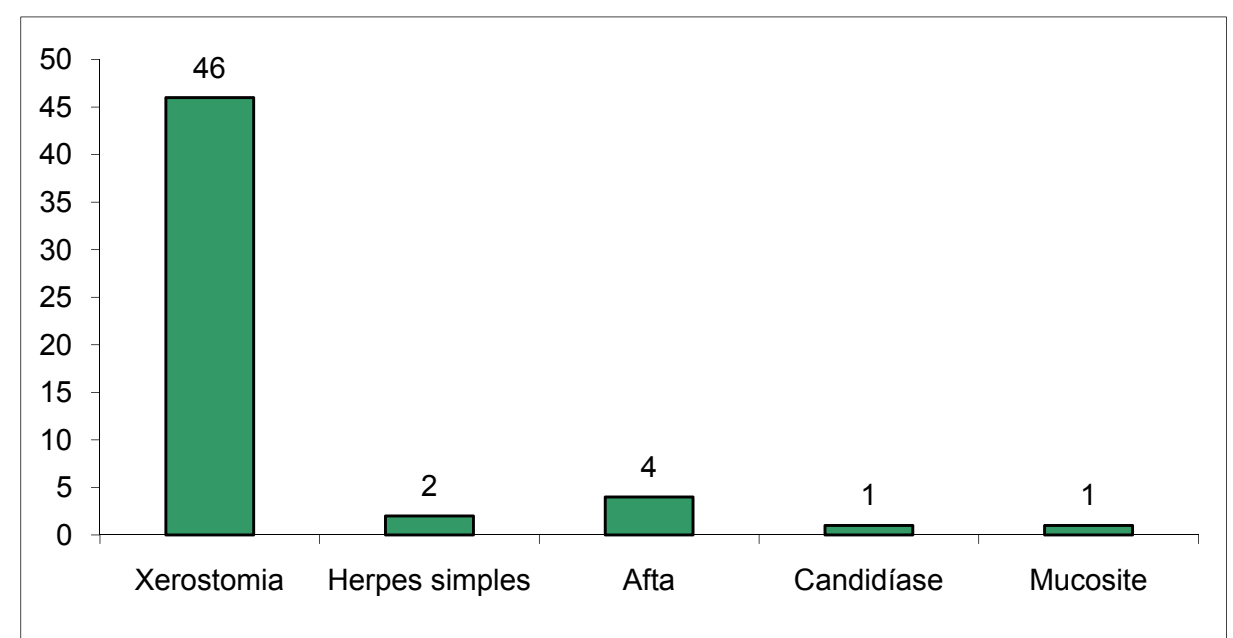

A quimioterapia do câncer destrói rapidamente as células proliferativas, mas também afeta células normais com alta taxa mitótica, principalmente as da mucosa oral e gastrointestinal e do sistema hematopoiético. ${ }^{1}$ Dreizen ${ }^{2}$ e Sung ${ }^{4}$ também relataram que nenhuma parte do corpo reflete as complicações do tratamento do câncer tão visivelmente quanto à boca. Do mesmo modo, Martins et al. ${ }^{9}$ explicaram que a ocorrência de lesões na cavidade oral é devido à alta sensibilidade dos tecidos e das estruturas orais aos efeitos tóxicos dos quimioterápicos.
Napeñas et al. ${ }^{18}$ afirmaram que a quimioterapia citotóxica compromete os mecanismos de defesa da boca fisicamente (danos diretos à mucosa) e imunologicamente (neutropenia) causando uma mudança na microflora oral.

Para manter a integridade dos tecidos orais, a saliva tem importantes funções como solução tampão, pH, defesa imunológica, proteção da mucosa e equilíbrio da microflora, além de outras funções, como fala, formação do bolo alimentar, deglutição e paladar. Quando um paciente é submetido à quimioterapia do câncer, ocorre uma diminuição 
do fluxo salivar, levando a um quadro conhecido como xerostomia, o que dificulta manter essas funções, podendo ocasionar o aumento do número de cáries devido ao acúmulo de bactérias e desenvolvimento de mucosite. 3, 9, 10,15,16

Martins et al. ${ }^{9}$ e Napeñas et al. ${ }^{18}$ relataram que a mucosite oral é um doloroso e debilitoso efeito causado pela citotoxicidade dos oncoterápicos e está presente entre $30 \%$ a $75 \%$ dos pacientes, dependendo da modalidade do quimioterápico. Vieira e Lopes ${ }^{17}$ afirmaram que seu aparecimento varia entre cinco a dez dias após o início da quimioterapia e na dependência do tipo de droga utilizada.

Mealey et al. ${ }^{3}$ afirmaram que a candidíase se desenvolve em pacientes que estão imunodeprimidos, assim como relataram que, além da candidíase, infecções recorrentes de herpes simples também são frequentemente reportadas em pacientes imunossuprimidos.

Os medicamentos quimioterápicos atuais disponíveis no mercado provocam muitos efeitos colaterais, podendo estar relacionados com complicações orais e produzir estomatotoxicidade direta ou indiretamente. A supressão da medula óssea produz trombocitopenia e neutropenia levando à diminuição da resistência a infecções. ${ }^{5}$ A Tabela 1 mostra a relação das manifestações orais encontradas nesta pesquisa com o quimioterápico utilizado pelos pacientes, na qual se observa que os medicamentos taxol e cisplatina, seguido do 5-fluorouracil foram os que mais provocaram alterações bucais. Essas drogas são utilizadas pela Unacon principalmente no tratamento dos cânceres de mama.

TABELA 1 - RELAÇÃO DAS MANIFESTAÇÕES ORAIS DE ACORDO COM O QUiMIOTERÁPICO UTILIZADO PELOS PACIENTES EM TRATAMENTO NA UNACON de ITAjaí SC.

\begin{tabular}{c|c|c|c}
\hline & Xerostomia & Infecções(candidíase,herpes, afta) & Mucosite \\
\hline Cisplatina & 11 & 2 & 1 \\
\hline Taxol & 10 & 2 & $\mathrm{X}$ \\
\hline 5-Fluorouracil & 7 & 2 & 1 \\
\hline Adriamicina & 6 & 1 & 1 \\
\hline Ciclofosfamida & 3 & 1 & 2 \\
\hline $\begin{array}{c}\text { Vincristina } \\
\text { (oncovin) }\end{array}$ & 2 & $\mathrm{x}$ & $\mathrm{X}$ \\
\hline
\end{tabular}

Alguns medicamentos são mais estomatotóxicos que outros, tais como o metotrexato, o 5-fluorouracil, o citarabine e a cisplatina, levando ao aparecimento de maior número de manifestações orais. Em relação à mucosite, uma das drogas que pode causá-la é o 5-fluorouracil, o qual, quando associado a outras drogas, tende a potencializar a ocorrência de mucosite. ${ }^{11,17,18}$

Para cada tipo de tumor existe um protocolo tanto de drogas quanto do número de ciclos; os efeitos colaterais orais estão relacionados com a dose da droga administrada e seu intervalo de tempo. Porém, nem todos os agentes quimioterápicos são igualmente estomatotóxicos ou causam os mesmo efeitos nos tecidos orais. ${ }^{12,16}$
O taxol foi o quimioterápico que apresentou maior número de manifestações orais, por ter sido o medicamento com maior frequência de aplicação principalmente pela sua indicação no tratamento do câncer de mama - o câncer encontrado com maior frequência neste trabalho.

Há que se observar que, em alguns pacientes com determinadas patologias, foi utilizado um regime quimioterápico associado por poliquimioterapia.

Este trabalho proporcionou conforto e sensação de bem-estar aos pacientes que estavam passando por um momento delicado em suas vidas. Já à Unacon, proporcionou a integração das áreas da saúde (odontologia, medicina e enfermagem), tratando o paciente, um indivíduo que necessita da atenção 
de todas as áreas concomitantemente, como um todo.

\section{Conclusão}

Após a análise dos resultados, concluise que: a maioria dos pacientes em tratamento quimioterápico apresentou algum tipo de manifestação oral; as alterações bucais mais encontradas foram a xerostomia, seguida de alterações no paladar (disgeusia) e infecções - as fúngicas (candidíase) e as virais (herpes simples recorrente) -; e houve maior incidência de pacientes do gênero feminino em tratamento quimioterápico e acima de 50 anos em ambos os gêneros.

\section{REFERÊNCIAS BibLIOGRÁFICAS}

1. Naylor GD, Terezhalmy GT. Oral complications of cancer chemotherapy: prevention and management. Spec Care Dent (Chicago) Jul- Aug 1988; 8(4):150-6.

2. Dreizen S. Oral complications of cancer therapies. Description an incidence of oral complications. NCI Monogr (Bethesda) 1990; 9:p.11-5.

3. Mealey BL, Semba SE, Hallmon WW. Dentistry and the cancer patient: oral manifestations and complications of chemotherapy. Compendium (Newtown) Oct 1994;15(10):1252-62.

4. Sung EC. Dental management of patients undergoing chemotherapy. J Calif Dent Assoc (San Francisco) Nov 1995; 23(11): 55-9.

5. Rosa LN. Atenção estomatológica aos pacientes pediátricos oncológicos. RGO (Porto Alegre) 1997 Mar- Apr; 45(2): 111-4.

6. Montenegro M, Franco M. Patologia: processos gerais. $4^{\mathrm{a}}$ ed. Rio de Janeiro: Atheneu; 1999.

7. Brasileiro Filho G. Bogliolo: Patologia. 7nd ed. Rio de Janeiro: Guanabara Koogan; 2000.

8. Castro RFM, Dezotti MSG, Azevedo LR de, Aquillante AG, Xavier CRG. Atenção odontológica aos pacientes oncológicos antes, durante e depois do tratamento antineoplásico. Rev
Odontol Unicid (São Paulo) Jan- Apr 2002; 14(1): 63-74.

9. Martins ACM, Caçador NP, Penteado W. Complicações bucais da quimioterapia antineoplásica. Acta Sci (Maringá) 2002; 24(3): 663-70.

10. Jensen SB, Pedersen A, Reibel J, Nauntofte B. Xerostomia and hypofunction of the salivary glands in cancer therapy. Support Care Cancer (Berlin) 2003; 11: 207-25.

11. Sandoval RL, Koga DH, Buloto LS, Suzuki K, Dib LL. Management of chemo- and radiotherapy induced oral mucositis with low-energy laser: initial results of A.C. Camargo Hospital. J Appl Oral Sci (Bauru) 2003; 11(4): 337-41.

12. Travaglini F. Complicações bucais no tratamento quimioterápico. 2006 Jan Available from: URL: <http://www. webodonto.com/html/artigo10.htm >.

13. Guerra MR, Gallo CV de M, Mendonça GA e S. Risco de câncer no Brasil: tendências e estudos epidemiológicos mais recentes. Rev Bras Cancerol (Rio de Janeiro) 2005; 51(3): 227-34.

14. Kumar V, Abbas AK, Fausto N. Patologia: Bases Patológicas das Doenças. $7^{\mathrm{a}}$ ed. Rio de Janeiro: Elsevier; 2005.

15. Mendonça EF de, Carneiro LS, Silva JB, Silva CM. Complicações bucais da quimioterapia e radioterapia no tratamento do câncer. Rev ABO Nac (São Paulo) Jun- Jul 2005; 13 (3):151-7.

16. Santos PS da, Fernandes KS. Complicações bucais da quimioterapia. 2006 Mar Available from: <http://www. abrale.org.br/apoio_profissional/artigos/complicacoes_bucais.php $>$.

17. Vieira ACF, Lopes FF. Mucosite oral: efeito adverso da terapia antineoplásica. Rev Ciênc Med Biol (Salvador) 2006; 5(3): 268-74.

18. Napeñas JJ, Shetty K, Bigler LR, Streckfus CF. Relationship between mucositis and changes in oral microflora during cancer chemotherapy. Oral Surg Oral Med Oral Pathol Oral Radiol Endod (St. Louis) Jan 2007; 103: 48-59.

Recebimento do artigo: 2/7/08 Aprovado: 25/12/08 